Tohoku J. Exp. Med., 2008, 214, 291-296

\title{
Increased Anxiety Level and High Salivary and Serum Cortisol Concentrations in Patients with Recurrent Aphthous Stomatitis
}

\author{
Eleni Albanidou-Farmaki, ${ }^{1}$ Athanasios K. Poulopoulos, ${ }^{1}$ Apostolos Epivatianos, ${ }^{1}$ \\ Konstantinos Farmakis, ${ }^{2}$ Michalis Karamouzis ${ }^{3}$ and Demetrios Antoniades ${ }^{1}$ \\ ${ }^{1}$ Department of Oral Medicine and Pathology, Dental School, Aristotle University of Thessaloniki, \\ Thessaloniki, Greece \\ ${ }^{2}$ Second Department of Surgery G., Gennimatas Hospital, Thessaloniki, Greece \\ ${ }^{3}$ Department of Biochemistry, AHEPA University Hospital, Thessaloniki, Greece
}

Recurrent aphthous stomatitis (RAS) is one of the most common oral mucosal disorders. The aim of the study was to determine any association between anxiety levels and concentrations of salivary and serum cortisol in patients with RAS. It has been suggested that stress with its presumed effects on the immune system, constitutes one of the major causative agents of RAS. The concentrations of salivary and serum cortisol were measured in 38 patients with recurrent aphthous stomatitis, and 38 healthy controls. Salivary and serum cortisol levels were measured using a Luminenscent Immunoassay (LIA) method. Anxiety levels were evaluated using Spielberger's State-Trait Anxiety Inventory which measures both trait anxiety as a general aspect of personality (STAI-T) and state anxiety as a response to a specific situation (STAI-S). The salivary cortisol levels were $1.44( \pm 0.58)$ $\mu \mathrm{g} \mathrm{dl}^{-1}$ in RAS patients and $0.91( \pm 0.56) \mu \mathrm{g} \mathrm{dl}^{-1}$ in controls $(p=0.001)$, while the serum cortisol levels were $3.13( \pm 1.59) \mu \mathrm{g} \mathrm{dl}^{-1}$ in RAS patients and $1.89( \pm 1.11) \mu \mathrm{g} \mathrm{dl}^{-1}$ in controls $(p=0.001)$. The state anxiety levels (STAI-S) were $48.85( \pm 9.7)$ in RAS group and $39.45( \pm 7.5)$ in control group $(p=0.001)$. The trait anxiety levels (STAI-T) were 49.78 $( \pm 13.02)$ in RAS group and $38.49( \pm 10.31)$ in control group $(p=0.001)$. Salivary and serum cortisol concentrations and state and trait anxiety levels in RAS were significantly higher than those in the control group. Our results suggest that stress may be involved in the pathogenesis of RAS. —— recurrent aphthous stomatitis; anxiety; salivary cortisol; serum cortisol; pathogenesis.

Tohoku J. Exp. Med., 2008, 214 (4), 291-296.

(C) 2008 Tohoku University Medical Press

Recurrent aphthous stomatitis (RAS) is one of the most common oral mucosal disorders. The reported prevalence in the general population varies from $5 \%$ to $66 \%$ with a mean of $20 \%$ (Ship 1996). The hypotheses of its pathogenesis are numerous. Several studies have reported a relationship between RAS and various causes but the results are conflicting (Scully 2006; Chattopadhyay and Chatterjee 2007). Different subgroups of patients appear to have different causes for the occurrence of RAS. These factors suggest a disease process that is triggered by a

Received May 14, 2007; revision accepted for publication February 4, 2008.

Correspondence: Dr. Eleni Albanidou-Farmaki, Assist. Prof., Department of Oral Medicine and Pathology, School of Dentistry, Aristotle University of Thessaloniki, 54006 Thessaloniki, Greece.

e-mail: elfarmaki@yahoo.gr 
variety of causative agents, each of which is capable of producing the disease in certain subgroups of patients (Porter et al. 1998). It is suggested that stress with its presumed effects on the immune system, constitutes one of the major causative agents of RAS (Barrons 2001). Previous studies report increased frequency of oral ulcer manifestation in intensely stressed populations (Soto Araya et al. 2004).

In conditions involving pain, anxiety, fright or acute tissue damage, many metabolic and endocrine changes occur, and among these a rise in the levels of blood cortisol is one of the most important physiological effects (Boudarene et al. 2002). Cortisol is a 21-carbon glucocorticoid secreted by the adrenal cortex with multiple biological effects, including the capability to affect the human stress response (Vedhara et al. 2003). The hypothalamus -pituitary-adrenal (HPA) axis becomes active in response to stress. Hence, increased levels of persistent anxiety problems have been associated with changes in HPA axis functioning and increased cortisol levels (Kudielka et al. 2004). Cortisol which is also called a stress hormone, has been used as an indicator in the stress evaluation studies (Takai et al. 2004). Cortisol is excreted primarily in urine in an unbound (free) form. Salivary cortisol measurement is reliable indicator of free cortisol or biologically active cortisol (Alpers et al. 2003), whereas serum cortisol measurement assesses total cortisol (bound and free) (Ritsner et al. 2007).

Cortisol production has an ACTH-dependent circadian rhythm with peak levels in the early morning and a minimum at night. The factors controlling this circadian rhythm are not completely defined. The circadian rhythm of ACTH/ cortisol secretion matures gradually during early infancy, and is disrupted in a number of physical and physiological conditions (Clow et al. 2004).

Stress has been suggested as a precipitating factor in RAS, as many patients made an association between RAS and what they term stress. In study groups of students, recurrences clustered around stressful periods of the academic year, conversely, periods of vacation were associated with a low frequency of lesions (Neville et al. 2002). Furthermore, it has been reported the association between stress and anxiety and saliva cortisol levels (Vedhara et al. 2003). A previous study investigated the possible association between anxiety, as measured by Hospital Anxiety and Depression scale (HAD), and salivary cortisol in patients with RAS, concluding that stress may play a role in the aetiology of RAS (McCartan et al. 1996).

Although stress and anxiety have been mentioned as possible factors related to development of RAS this association somewhat remains controversial. The aim of this study was to assess the association between anxiety and cortisol in serum and saliva, in patients with RAS by using both psychological testing instrument (State-Trait Anxiety Inventory [STAI]) and physiological testing instrument (salivary-serum cortisol).

\section{Patients and Methods}

The study group comprised of 38 RAS patients (21 females, 17 males, mean age $=37 \pm 4$ years, S.D. $=17.56$ ) The control group composed of 38 healthy individuals ( 22 females, 16 males, mean age $=32 \pm 7$ years, s.D. $=$ 13.78). All experiments were performed in compliance with the relevant laws and our institutional guidelines. Informed consent was obtained from the patients which participated in the study.

Collection of blood and saliva was performed approximately the same time (10-10.30 a.m.) in all patients, and was established nearly the same awakening time for all the patients, since it is known that cortisol levels in blood serum and consequently in the saliva is not stable during the course of the day. None of the patients and the control group had any systemic disease, including diseases of the endocrine or metabolic systems, or hematological deficiencies, nor were they using any corticosteroids or other drugs such as oral contraceptives, and all were non-smokers.

Blood was centrifuged at 2,500 rpm/min. Blood serum was collected and stored at $-20^{\circ} \mathrm{C}$ until use. Saliva collection proceeded stimulation of patients via administration of $1 \mathrm{ml}$ of citric acid $10 \%$. Saliva was also centrifuged at 2,500 $\mathrm{rpm} / \mathrm{min}$ in order to precipitate any mucus present. The supernatant was collected and stored at $-20^{\circ} \mathrm{C}$ until use.

Saliva and serum cortisol was measured by 
Luminenscent Immunoassay (LIA) using the Vitros Cortisol Reagent Pack and Vitros immunodiagnostic Products Cortisol Calibrators on the Vitros Immunodiagnostic System (Ortho-Clinical Diagnostics Inc., Rochester, NY, USA). A competitive immunoassay technique is used, which depends on the competition between cortisol present in the sample with a horseradish peroxidase (HRP)-labeled cortisol conjugate for a limited number of binding sites on a biotinylated antibody (sheep anti-cortisol). The antigen-antibody complex is captured by streptavidin on the wells, unbound materials are removed washing. The bound HRP conjugate is measured by a luminescent reaction (Westermann et al. 2004). A reagent containing luminogenic substrates (a luminol derivative and a peracid salt) and an electron transfer agent, is added to the wells.

The HRP in the bound conjugate catalyzes the oxidation of the luminol derivative, producing light. The electron transfer agent (a substituted acetanilide) increases the level of light produced and prolongs its emission. The light signals are read by the Vitros System. The amount of HRP conjugate bound is indirectly proportional to the concentration of cortisol present. The intra- and the interassay variation coefficients were less than $5.0 \%$ for both serum and saliva cortisol at the level of 146 $\mathrm{nmol} / \mathrm{l}$, and the detection limit was $3 \mathrm{nmol} / \mathrm{l}$.

Anxiety levels were measured by Spielberger's STAI (Spielberger et al. 1983) which measures both trait anxiety as a general aspect of personality (STAI-T) and state anxiety as a response to a specific situation (STAI-S). A person can experience an emotional status evoked by a condition is considered STAI-S, which is transitory. STAI-T refers to relatively enduring individual differences in anxiety proneness, such as diathesis of emotional vulnerability, which accounts for differences between people in the tendency to perceive stressful situations as dangerous or threatening and to respond to such situations with an increase in intensity of their STAI-S. The sensitivity of STAI-S and STAI-T scale to external stress has been shown repeatedly in research on emotional reactions. Another unique feature of STAI is its purer measure of anxiety independent of depression. STAI consists of 40 statements, 20 of which measure trait anxiety and 20 state anxiety. Items are scored on 4 point scales, with response categories varying according to the nature of the question. For both levels the range of values is between 20 and 80 and the higher point scored the higher the level of anxiety. The above mentioned questionnaire has been tested extensively for reliability and validity (Spielberger et al. 1983).
$T$-test was used to compare the trait, state anxiety and salivary and serum cortisol levels between patients with RAS and the control group. Pearson's correlation analysis was used to study the correlation among the above mentioned variables in patients with RAS. Logistic regression analysis was used to evaluate the variables related with RAS. Logistic regression analysis was used for calculation of each variable's independent contribution to dependent variable. Dependent variable must always be dichotomous, as group membership. RAS was a dependent variable, and salivary, serum, state and trait anxiety levels were independent variables in this model.

\section{RESUlts}

The state and trait anxiety and cortisol serum and salivary levels of 76 subjects were analysed. Patients' characteristics and demographic data are summarized in Table 1.

The salivary cortisol levels were 1.44 $( \pm 0.58) \mu \mathrm{g} \mathrm{dl}^{-1}$ in RAS patients and $0.91( \pm 0.56)$ $\mu \mathrm{g} \mathrm{dl}^{-1}$ in controls $(p=0.001)$, while the serum cortisol levels were $3.13( \pm 1.59) \mu \mathrm{g} \mathrm{dl}^{-1}$ in RAS patients and $1.89( \pm 1.11) \mu \mathrm{g} \mathrm{dl}^{-1}$ in controls $(p=$ $0.001)$ as indicated in Table 2 . The state anxiety levels (STAI-S) in RAS group were $48.85( \pm 9.7)$ and $39.45( \pm 7.5)$ in control group $(p=0.001)$ (Table 2). The trate anxiety levels (STAI-T) in RAS group were $49.78( \pm 13.02)$ and 38.49 $( \pm 10.31)$ in control group $(p=0.001)$ (Table 2). In all, $59 \%$ of patients in RAS group had a STAI-S score $>40$ and $62 \%$ of patients in RAS group had a STAI-T $>40$.

We found that salivary and serum cortisol, state and trait anxiety levels in RAS group were significantly higher than control group. There was a positive correlation between STAI-S and STAI-T scores $(r=0.482, p=0.003$ in control

TABLE 1. Patients characteristics.

\begin{tabular}{lcc}
\hline & $\begin{array}{c}\text { Study group } \\
(n=38)\end{array}$ & $\begin{array}{c}\text { Control group } \\
(n=38)\end{array}$ \\
\hline Females & 21 & 22 \\
Males & 17 & 16 \\
Mean age & $37 \pm 4$ & $32 \pm 7$ \\
S.D. & 17.56 & 13.78 \\
\hline
\end{tabular}


TABLE 2. Salivary and serum cortisol levels, STAI-S and STAI-T scores (mean \pm S.D.) of patients.

\begin{tabular}{lcccc}
\hline & $\begin{array}{c}\text { Study group RAS patients } \\
(n=38)\end{array}$ & $\begin{array}{c}\text { Control group } \\
(n=38)\end{array}$ & $t$-value & $p$-value \\
\hline Salivary cortisol & $1.44 \pm 0.58 \mu \mathrm{g} \mathrm{dl}^{-1}$ & $0.91 \pm 0.56 \mu \mathrm{g} \mathrm{dl}^{-1}$ & 4.09 & 0.001 \\
Serum cortisol & $3.13 \pm 1.59 \mu \mathrm{g} \mathrm{dl}^{-1}$ & $1.89 \pm 1.11 \mu \mathrm{g} \mathrm{dl}^{-1}$ & 4.36 & 0.001 \\
State anxiety & $48.85 \pm 9.7$ & $39.45 \pm 7.5$ & 4.82 & 0.001 \\
Trait anxiety & $49.78 \pm 13.02$ & $38.49 \pm 10.31$ & 4.19 & 0.001 \\
\hline
\end{tabular}

$* t$-test.

STAI, state-trait anxiety inventory.

TABLE 3. Logistic regression analysis, in which the group membership (RAS or control) was dependent variable.

\begin{tabular}{lcccc}
\hline \multicolumn{1}{c}{ Variable } & B & Wald & d.f. & $p$-value \\
\hline Salivary cortisol & -1.69 & 9.33 & 1 & 0.02 \\
Serum cortisol & -1.73 & 9.27 & 1 & 0.02 \\
STAI-S & -0.09 & 3.95 & 1 & 0.04 \\
STAI-T & -0.06 & 4.61 & 1 & 0.03
\end{tabular}

STAI, state-trait anxiety inventory.

group and $r=0.412, p=0.008$ in study group) There was no correlation between saliva cortisol levels and neither STAI-S ( $r=0.06$ RAS group, $r=0.07$ control group) nor STAI-T ( $r=0.01$ RAS group, $r=0.22$ control group) in both group. Furthermore no correlation was found between serum cortisol levels and neither STAI-S $(r=0.1$ RAS group, $r=0.2$ control group) nor STAI-T ( $r=0.02$ RAS group, $r=0.4$ control group) in both group.

A logistic regression model in which the RAS was dependent variable and salivary cortisol levels, serum cortisol levels, STAI-S, STAI-T were taken as independent variables was performed. Salivary cortisol levels $(B=-1.69$, wald $=9.33$, d.f. $=1, p=0.02$ ), serum cortisol levels $(\mathrm{B}=-1.73$, wald $=9.27$, d.f. $=1, p=0.02)$, STAI-S $(\mathrm{B}=-0.09$, wald $=3.95$, d.f. $=1, p=0.04)$ and STAI-T $(\mathrm{B}=-0.06$, wald $=4.61$, d.f. $=1, p=$ $0.03)$ scores were found significantly related with RAS. Group membership of each individual can be predicted correctly with $76.4 \%$ probability in this model (Table 3).

\section{Discussion}

The aetiopathogenesis of RAS appears to be complex, interactions with genetic, nutritional, and hematologic factors are reported (Rogers 1997; Saral et al. 2005). Much has now been clarified about the mechanisms involved, and interesting new associations such as the involvement of a $\mathrm{T}$ cell mediated immunologic reaction have emerged (Natah et al. 2000). The aim of the present study was to determine the association between anxiety and cortisol in serum and saliva, in patients with RAS by using both psychological testing instrument (STAI) and physiological testing instrument (salivary -serum cortisol).

Stress has been postulated as a precipitating factor in RAS. However has been reported little objective evidence to support such an association (Peterson 1989). Nevertheless, many patients make an association between RAS and what they term stress. It has been reported that previous studies have focused on individuals who in retrospect were more likely to have had trait anxiety as a basis for their perceived stress (McCartan et al. 1996). Trait anxiety is nearly equivalent to stress 
and can be measured, although the biochemical response to both may differ. An insight into patient's psychological status can be estimated from both serum free and salivary cortisol levels (Vedhara et al. 2003; Takai et al. 2004).

Mental and physical stress have both been studied in relation to cortisol, and both forms of stress increased cortisol levels but only transiently (Schommer et al. 2003). Whereas anxiety is a definable psychosocial entity which is manifest as an exaggerated response to a given situation (as in state anxiety) or present all the time as a behavioral characteristic (as in trait anxiety). There is good evidence that stress and anxiety are related to increased resting levels of cortisol (Schommer et al. 2003; Wirtz et al. 2007).

A previous study investigated the possible association between anxiety, as measured by Hospital Anxiety and Depression scale (HAD), and salivary cortisol in patients with RAS, concluding that stress may play a role in the etiology of RAS (McCartan et al. 1996).

Our study used for the evaluation of anxiety the Spielberger's STAI, a more sensitive method, which measures both trait anxiety as a general aspect of personality (STAI-T) and state anxiety as a response to a specific situation (STAI-S), whereas McCartan et al. (1996) used the HAD scale, which was designed to assess only trait anxiety in the recent past. In our study the study groups were carefully selected by excluding patients with hematological deficiency states (such as iron deficiency and vitamin B12 deficiency), whereas in McCartan's study one study group was composed of patients with detected hematological deficiencies. Although our study design was different, the salivary cortisol levels in our patients with RAS were comparable with those reported by McCartan et al. (1996).

Studies in humans where cortisol levels were measured in both blood serum and saliva of RAS patients have given equivalent values (Harris et al. 1989). The measurement of cortisol in saliva provides several advantages over its measurement in serum or plasma. Sample collection for salivary cortisol measurement is non-invasive and inexpensive, and specimen collection is easy to perform in infants and children (Schmidt 1998). The former view is also supported by the fact that cortisol levels in the saliva are not affected by plaque or gingival gum inflammation (Kurer et al. 1995).

A variety of mechanisms may be considered as related between stress and recurrent oral ulcers. We may assume that an unknown biochemical reaction takes place or stress could possibly lead to atypical habits that injure the oral mucosa, such as bites in cheek and lips, leading to oral ulcer manifestation (Paterson et al. 1995).

Our study reveals a positive correlation between RAS and both state and trait anxiety; therefore it is not possible to distinguish the effect of the two components of anxiety separately. As a consequence we cannot declare whether the RAS lesions are more related to state or to trait anxiety: however this does not play an important role in the psychological treatment of the patient as the management of stress is based on reducing both components of anxiety.

This study provides evidence for trait anxiety being a feature of some patients with RAS. Patients who are not normal from a trait anxiety point of view appear to have greater levels of anxiety and salivary and serum cortisol levels at the time of their ulceration. The mechanisms by which trait anxiety predisposes to RAS remains to be elucidated, therefore may have implication for the management of RAS.

In the present study, we were able to determine a positive association between elevated cortisol levels in the blood serum and saliva, and state and trait anxiety in RAS patients. Therefore, measurement of the salivary, serum cortisol, and state and trait anxiety which reflect response to stress, seems a promising parameter in the investigation of RAS. In conclusion, we suggest that RAS patients may need supportive phychological management to increase their ability to cope with stress together with the conventional treatment methods.

\section{References}

Alpers, G.W., Abelson, J.L., Wilhelm, F.H. \& Roth, W.T. (2003) Salivary cortisol response during exposure treatment in 
driving phobics. Psychosom. Med., 65, 679-687.

Barrons, R.W. (2001) Treatment strategies for recurrent oral aphthous ulcers. Am. J. Health Syst. Pharm., 58, 41-50.

Boudarene, M., Legros, J.J. \& Timsit-Berthier, M. (2002) Study of the stress response: role of anxiety, cortisol and DHEAs. Encephale, 28, 139-146.

Chattopadhyay, A. \& Chatterjee, S. (2007) Risk indicators for recurrent aphthous ulcers among adults in the US. Community Dent. Oral Epidemiol., 35, 152-159.

Clow, A., Thorn, L., Evans, P. \& Hucklebridge, F. (2004) The awakening cortisol response : Methodological issues a significance. Stress, 7, 29-37.

Harris, B., Cook, N.J., Walker, R.F., Read, G.F. \& Riad-Fahmy, D. (1989) Salivary steroids and psychometric parameters in male marathon runners. Br. J. Sports Med., 23, 89-93.

Kudielka, B.M., Buske-Kirschbaum, A., Hellhammer, D.H. \& Kirschbaum, C. (2004) HPA axis responses to laboratory psychosocial stress in healthy elderly adults, younger adults, and children: impact of age and gender. Psychoneuro-Endocrinol., 29, 83-98.

Kurer, J.R., Watts, T.L., Weinman, J. \& Gower, D.B. (1995) Psychological mood of regular dental attenders in relation to oral hygiene behaviour and gingival health. J. Clin. Periodontol., 22, 52-55.

McCartan, B.E., Lamey, P.-J. \& Wallace, A.M. (1996) Salivary cortisol and anxiety in recurrent apthous stomatitis. J. Oral Pathol. Med., 25, 357-359.

Natah, S.S., Hayrinen-Immonen, R., Hietanen, J., Patinen, P., Malmstrom, M., Savilahti, E. \& Konttinen, Y.T. (2000) Increased density of lymphocytes bearing gamma/delta T-cell receptors in recurrent aphthous ulceration (RAU). Int. J. Oral Maxillofac. Surg., 29, 375-380.

Neville, B.W., Damm, D.D., Allen, C.M. \& Bouquot, J.E. (2002) Oral and Maxillofacial Pathology 2nd., WB Saunders Co., Philadelphia.

Paterson, A.J., Lamb, A.B., Clifford, T.J. \& Lamey, P.-J. (1995) Burning mouth syndrome: the relationship between the HAD scale and paratunctional habits. J. Oral Pathol. Med., 24, 289-292.

Peterson, A. (1989) Psychological stress and recurrent aphthous ulceration. J. Oral Pathol. Med., 18, 119-123.

Porter, S.R., Scully, C. \& Pedersen, A. (1998) Recurrent aphthous stomatitis. Crit. Rev. Oral Biol. Med., 9, 306-321.

Ritsner, M.S., Gibel, A., Ponizovsky, A.M., Shinkarenko, E., Ratner, Y. \& Kurs, R. (2007) State and trait related predictors of serum cortisol to DHEA(S) molar ratios and hormone concentrations in schizophrenia patients. Eur. Neuropsycho-Pharmacol., 17, 257-264.

Rogers, R.S. (1997) Recurrent aphthous stomatitis : clinical characteristics and associated systemic disorders. Semin. Cutan. Med. Surg., 16, 278-283.

Saral, Y., Coskun, B.K., Ozturk, P., Karatas, F. \& Ayar, A. (2005) Assessment of salivary and serum antioxidant vitamins and lipid peroxidation in patients with recurrent aphthous ulceration. Tohoku J. Exp. Med., 206, 305-312.

Schmidt, N.A. (1998) Salivary cortisol testing in children. Issues Compr. Pediatr. Nurs., 20, 183-190.

Schommer, N.C., Hellhammer, D.H. \& Kirschbaum, C. (2003) Dissociation between reactivity of the hypothalamuspituitary-adrenal axis and the sympathetic-adrenalmedullary system to repeated psychosocial stress. Psychosom. Med., 65, 450-460.

Scully, C. (2006) Clinical practice. Aphthous ulceration. $N$. Engl. J. Med., 355, 165-172.

Ship, J.A. (1996) Recurrent aphthous stomatitis. An update. Oral Surg. Oral Med. Oral Pathol. Oral Radiol. Endod., 81, 141-147.

Soto Araya, M., Rojas Alcayaga, G. \& Esguep, A. (2004) Association between psychological disorders and the presence of Oral lichen planus, Burning mouth syndrome and Recurrent aphthous stomatitis. Med. Oral, 9, 1-7.

Spielberger, C., Gorsuch, R. \& Lushene, R. (1983) STAI Manual for the State-Trait Anxiety Inventory. Palo Alto: Calif. Consulting Psychologists Press.

Takai, N., Yamaguchi, M., Aragaki, T., Eto, K., Uchihashi, K. \& Nishikawa, Y. (2004) Effect of psychological stress on the salivary cortisol and amylase levels in healthy young adults. Arch. Oral Biol., 49, 963-968.

Vedhara, K., Miles, J., Bennett, P., Plummer, S., Tallon, D., Brooks, E., Gale, L., Munnoch, K., Schreiber-Kounine, C., Fowler, C., Lightman, S., Sammon, A., Rayter, Z. \& Farndon, J. (2003) An investigation into the relationship between salivary cortisol, stress, anxiety and depression. Biol. Psychol., 62, 89-96.

Westermann, J., Demir, A. \& Herbst, V. (2004) Determination of cortisol in saliva and serum by a luminescence-enhanced enzyme immunoassay. Clin. Lab., 50, 11-24.

Wirtz, P.H., Elsenbruch, S., Emini, L., Rudisuli, K., Groessbauer, S. \& Ehlert, U. (2007) Perfectionism and the cortisol response to psychosocial stress in men. Psychosom. Med., 69, 249-255. 